\title{
science \\ Open access status of journals and articles in Journal Citation Reports
}

\author{
Sang-Jun Kim', Kay Sook Park ${ }^{2}$ \\ Library, ${ }^{1}$ Korea Research Institute of Bioscience and Biotechnology, ${ }^{2}$ Electronics and Telecommunications Research Institute, \\ Daejeon, Korea
}

\section{Abstract}

Purpose: There is somewhat of a difference between understanding the open access (OA) concept and practicing it by stakeholders. OA articles are mainly published by gold and hybrid OA journals, but the OA status may be confusing depending on the target databases. This study investigated the OA status of journals and articles and evaluated the extent to which OA2020 (publishing 90\% of articles as OA) was achieved.

Methods: This study collected OA data by combining 2014-2019 data from Journal Citation Reports at the journal level with Web of Science at the article level. Finally, 12,449 journals were analyzed focusing on gold and hybrid OA journals, and progress towards the goal of OA2020 was evaluated.

Results: Even though 80.4\% of Journal Citation Reports journals were gold and hybrid OA journals, only $20.9 \%$ of the articles were OA (gold OA journals, $16.6 \%$; hybrid journals, $4.3 \%$ ). The compound annual growth rate of the total articles was $4.7 \%$, that of OA articles was $16.4 \%$, and that of subscription articles was only $1.7 \%$. Among the subscription journals, $77.4 \%$ had shifted to become hybrid journals, but only $5.2 \%$ of their articles were OA. Therefore, the hybrid journals were at the very early stage of $\mathrm{OA}$ publishing.

Conclusion: Considerable progress must still be made to achieve the goal of OA2020. The influence of OA publishing will eventually expand and therefore, librarians should take interest in OA publishing for the library services.

Keywords

Received: October 7, 2020

Accepted: December 5, 2020

Article processing charge; Gold open access journal; Hybrid journal; Journal Citation Reports; Open access

Correspondence to Sang-Jun Kim sjkim@kribb.re.kr

ORCID

Sang-Jun Kim

https://orcid.org/0000-0002-1833-2236

Kay Sook Park

https://orcid.org/0000-0002-2771-4930

\section{Introduction}

Background/rationale: Competitive research activities and computer-aided publishing technology have led to explosive growth in e-journals over the past 20 years. This has led to steady increases in libraries' budgets for journal subscriptions. The digital divide in e-journals has 
resulted in the emergence of the open access (OA) concept, which allows anyone to freely access and reuse articles without any barriers. While print journals usually have limitations in the number of articles for each issue, there is no such limitation in e-journals, which are distributed on the internet. Therefore, e-journal publishers introduced a new publishing model, wherein an article processing charge (APC) is charged for authors instead of a journal subscription fee for readers. In APC-based OA publishing, the author owns the copyright for the article by paying an APC to the publisher directly, and determines the terms of use by applying a Creative Commons License. OA publishing is a new business model, and the global OA movement is developing in complex and diverse ways. Therefore, it is necessary to assess the progress that has been made towards achieving the goal of OA2020, which aims to convert more than $90 \%$ of the articles annually published worldwide (about 2 million) to OA [1].

As shown in Fig. 1, there are several variants of OA types at the journal level or the article level. Journal-based OA types can be divided into hybrid OA and gold OA, while articlebased OA types are black, green, bronze, and gold OA. In an analysis of the status of OA articles as of June 2020 in Dimensions, Scopus, and Web of Science (WoS), gold and bronze OA articles comprised 18.4\%, 13.7\%, and 15.9\% of articles, respectively. Most subscription journals are currently hybrid journals with an optional model in which the author can convert an article to OA by paying an APC. Therefore, hybrid journals are a mixture of open articles and closed articles. There are two types of gold OA journals: gold OA journals without APCs, which are mainly published by non-profit organizations that cover all the publication costs, and gold OA journals with APCs, where the authors bear the costs of APCs for each article.

Each bibliographic database contains articles with different document types, and there are also generally differences among databases in terms of the evaluation criteria for journals and the classification of OA types. Consequently, it is difficult to determine how global OA status should be assessed in terms of OA type and level. Some studies have investigated the status of OA publishing [2], but have not provided reliable information on OA status. Scopus does not subdivide its content by OA types, whereas WoS defines OA types at the journal and article levels. Journal Citation Reports (JCR) classifies journals by OA type, but it only provides the number of OA articles for the last 3 years and its data cannot be downloaded. Therefore, combining data from WoS at the article level and JCR at the journal level was chosen as the most appropriate method for reliable research on the current status of OA publishing.

Objectives: Librarians in charge of journal and article services need to grasp the current situation regarding OA accurately, and then prudently predict and prepare for the future of academic publishing and journal subscription. OA articles are mainly published in gold and hybrid OA journals. This study investigated the transition status of traditional subscription journals to the OA publishing model as well as the current status of gold OA journals. It also focused on their growth rate and the ratio of subscription articles to $\mathrm{OA}$ articles. We also analyzed the largest existing publishers, along with new OA publishers. This study's ultimate objective was to evaluate the degree to which the goal of OA2020 has been achieved in JCR and to explore its feasibility for libraries facing the serials crisis. These results will provide useful information for librarians regarding $\mathrm{OA}$ status.

\section{Methods}

Ethics statement: This was not a study with human subjects, so neither institutional review board approval nor informed consent was required.

Study design: This was a literature database-based descriptive study.

Data collection and analysis: As this study aimed to assess the OA publication status from the library budget perspective,

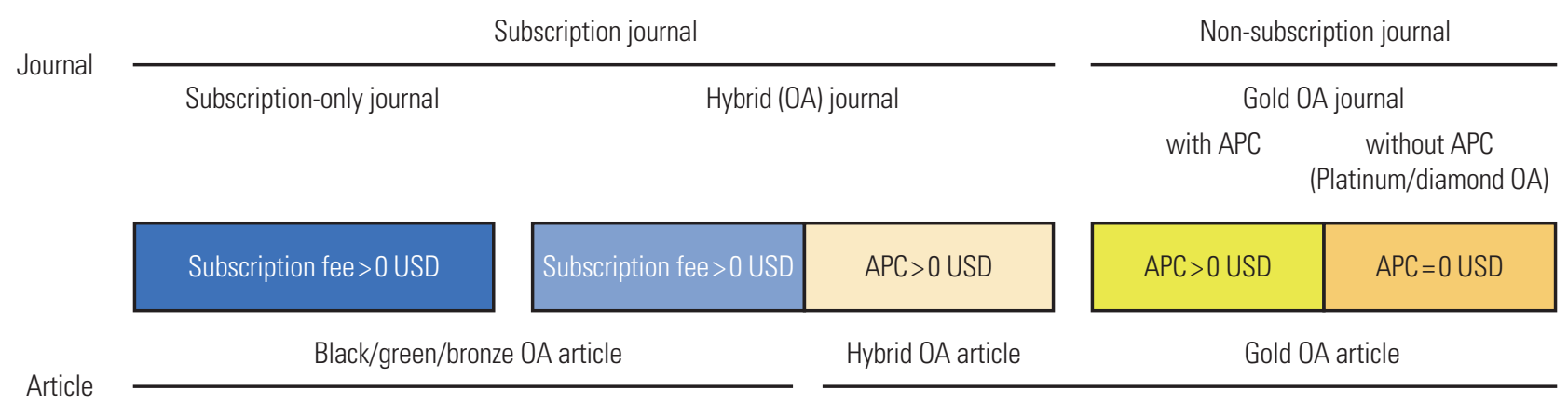

Fig. 1. Open access $(O A)$ types for journals and articles used in this study. APC, article processing charge; USD, US dollars. 
gold and hybrid OA journals were analyzed at the journal level. JCR data from 2014 to 2019 and WoS were used to obtain up-to-date and exact information on OA status (Dataset 1). To obtain information about OA status, WoS was searched in June 2020 for SCIE and SSCI articles with the queries of "document type is article or review" and "year range is from 2014 to 2019 ," which yielded 9,959,063 articles in 12,561 journals. Subsequently, the search results were limited by the OA type as "Directory of Open Access Journals (DOAJ)-Gold" and "Other Gold", and were downloaded by year. The WoS data on OA articles were integrated with the JCR journal list. In this process, 1,600 journals whose journal names did not match each other were investigated manually. However, 69 journals not contained in the JCR journal list and 96 journals with no articles in WoS were excluded. Finally, 9,575,780 articles in 12,449 journals were acquired, and this study analyzed $96 \%$ of the articles in the results of the WoS search. Therefore, there was no significant difference between this study, which combined JCR and WoS, and a study design that would be fully based on WoS.

In order to analyze the OA status more precisely, the OA type of journals based on WoS was redefined in this study. First, "DOAJ-Gold" journals were classified as gold OA journals and "Other Gold" as hybrid journals. Subsequently, gold OA journals were divided into gold OA journals without APCs and gold OA journals with APCs. The journals for which the APC was listed as free in DOAJ and determined to be 0 USD in the author's previous study [3] were classified as gold OA journals without APCs, and the rest as gold OA journals with APCs. The final judgment was made through a comprehensive consideration of 6 years of OA articles in WoS and 3 years of OA articles in JCR. In this study, the number of articles per journal was based on the citable items in JCR 2014 to 2019, while the number of OA articles in each hybrid journal was determined by applying the search results of WoS.

Statistical methods: This study was based on gold and hybrid OA journals and only descriptive statistics were presented. Data were tabulated and the proportions of the cells were calculated. The compound annual growth rate (CAGR) was calculated.

\section{Results}

\section{The OA journals and articles in JCR}

The classification results of the JCR 2014 to 2019 journals by OA type are shown in Table 1. Gold OA journals accounted for only $13.2 \%$ of journals, while hybrid and subscription-only journals accounted for $67.2 \%$ and $19.6 \%$, respectively. Regardless of the number of $\mathrm{OA}$ articles, OA publishing was an option for $80.4 \%$ of JCR journals, while only $19.6 \%$ strictly
Table 1. OA journals and articles in Journal Citation Reports 2014 to 2019

\begin{tabular}{lccccc}
\hline \multirow{2}{*}{ Journal type } & \multicolumn{2}{c}{ Journal } & & \multicolumn{2}{c}{ Article } \\
\cline { 2 - 3 } \cline { 5 - 6 } & Number & Rate (\%) & & Number & Rate (\%) \\
\hline Gold OA & & & & & \\
without APC & 317 & 2.6 & & 129,998 & 1.4 \\
with APC & 1,323 & 10.6 & & $1,457,004$ & 15.2 \\
Hybrid & 8,369 & 67.2 & & $412,860^{\text {a) }}$ & 4.3 \\
& & & & $6,612,719^{\text {b) }}$ & 69.0 \\
Subscription & 2,440 & 19.6 & & 963,199 & 10.1 \\
Total & 12,449 & 100.0 & & $9,575,780$ & 100.0 \\
\hline
\end{tabular}

$\mathrm{OA}$, open access; $\mathrm{APC}$, article processing charge.

${ }^{a} \mathrm{OA}$ articles in the hybrid journals; ${ }^{b}$ Subscription articles in the hybrid journals.

adhered to a subscription model. The gold OA journals were divided into 317 gold OA journals without APCs and 1,323 gold OA journals with APCs. The hybrid journals were subdivided into a large group of 4,462 journals that had published more than 12 OA articles over the course of 6 years and a small group of 3,907 journals that had published fewer than $12 \mathrm{OA}$ articles. However, in the former group, the average number of OA articles per journal was only 15 articles annually. The latter group comprised hybrid journals in terms of the APC and the journal price in the previous study [3], but 589 of these journals had not published any OA articles in 6 years. Thus, most of the hybrid journals were at the early stage of OA publishing.

In the JCR 2014 to 2019, the total number of OA articles reached about 2 million, accounting for $20.9 \%$ of all articles (16.6\% from gold OA journals and 4.3\% from hybrid journals). In a comparison limited to the gold and hybrid OA types, the ratio of OA journals was 16:84, but that of OA articles was 79:21, showing the opposite tendency. Therefore, the gold OA journals published about four times more OA articles than the hybrid journals, and the majority of OA articles were from the gold OA journals.

\section{Annual growth of OA journals and articles}

The share of OA articles increased from 16.0\% in JCR 2014 to $27.1 \%$ in JCR 2019. Over the past 6 years, the total number of articles increased at a CAGR of $4.7 \%$. The CAGR of OA articles was $16.4 \%$, while that of subscription articles was $1.7 \%$, including hybrid and subscription-only journals (Table 2). Therefore, OA articles have contributed significantly to the growth of WoS and JCR. The gold OA journals increased more than the hybrid journals, but the subscription-only journals decreased. Finally, the total number of JCR journals increased at a CAGR of $1.8 \%$. The CAGR of the OA articles was higher in the hybrid journals than in the gold OA jour- 
Table 2. Annual OA journals and articles in Journal Citation Reports 2014 to 2019

\begin{tabular}{|c|c|c|c|c|c|c|c|c|}
\hline Item & OA type & 2014 & 2015 & 2016 & 2017 & 2018 & 2019 & $\operatorname{CAGR}(\%)$ \\
\hline \multirow{3}{*}{ Journal } & Gold OA with APC & 904 & 943 & 993 & 1,078 & 1,157 & 1,305 & 7.6 \\
\hline & Hybrid & 7,666 & 7,767 & 7,874 & 7,999 & 8,124 & 8,245 & 1.5 \\
\hline & Subscription & 2,266 & 2,168 & 2,209 & 2,225 & 2,213 & 2,214 & -0.5 \\
\hline \multirow{5}{*}{ Article } & Gold OA with APC & 172,044 & 197,042 & 219,290 & 236,280 & 282,458 & 349,890 & 15.3 \\
\hline & Hybrid-OA & 37,239 & 45,143 & 63,043 & 69,796 & 82,952 & 114,687 & 25.2 \\
\hline & Subtotal & 229,160 & 263,301 & 302,746 & 328,062 & 388,524 & 489,069 & 16.4 \\
\hline & Share $(\%)^{b)}$ & $(16.0)$ & $(17.7)$ & $(19.6)$ & $(20.5)$ & (22.8) & $(27.1)$ & - \\
\hline & Total & $1,434,686$ & $1,491,085$ & $1,544,151$ & $1,597,875$ & $1,705,575$ & $1,802,408$ & 4.7 \\
\hline
\end{tabular}

$\mathrm{OA}$, open access; $C A G R$, compound annual growth rate; $\mathrm{APC}$, article processing charge.

a) Hybrid-OA: OA articles in the hybrid journals; ${ }^{\text {bl} S h a r e: ~ O A ~ a r t i c l e s ~ a m o n g ~ t h e ~ t o t a l ; ~}{ }^{c l}$ Hybrid-subscription: subscription articles in the hybrid journals.

Table 3. OA journals and articles of the 10 major publishers in Journal Citation Reports 2014 to 2019

\begin{tabular}{|c|c|c|c|c|c|c|c|c|c|c|c|c|c|c|}
\hline OA type & & \multicolumn{6}{|c|}{ Top five subscription publisher } & \multicolumn{6}{|c|}{ Top five OA publisher } & Total \\
\hline \multirow[t]{3}{*}{ Journal } & Gold OA & 104 & 97 & 65 & 3 & 66 & 335 & 71 & 226 & 7 & 43 & 70 & 417 & 752 \\
\hline & Hybrid & 1,392 & 1,675 & 1,160 & 41 & 1,047 & 5,315 & - & 4 & - & - & 3 & 7 & 5,322 \\
\hline & Total & 1,496 & 1,772 & 1,225 & 44 & 1,113 & 5,650 & 71 & 230 & 7 & 43 & 73 & 424 & 6,074 \\
\hline & Total & 257,467 & 142,660 & 91,003 & 73,111 & 39,136 & 603,377 & 210,910 & 167,271 & 148,196 & 97,691 & 82,791 & 706,859 & $1,310,236$ \\
\hline & Share $(\%)^{b)}$ & 12.9 & 7.1 & 4.6 & 3.7 & 2.0 & 30.2 & 10.5 & 8.4 & 7.4 & 4.9 & 4.1 & 35.3 & 65.5 \\
\hline & CAGR (\%) & 21.3 & 19.1 & 43.3 & -4.5 & 26.8 & 20.8 & 58.4 & 6.4 & -15.4 & 35.7 & 1.5 & 16.1 & 18.0 \\
\hline
\end{tabular}

OA, open access; RSC, Royal Society of Chemistry; T\&F, Taylor \& Francis; BMC, BioMed Central; PLoS, Public Library of Science; CAGR, compound annual growth rate.

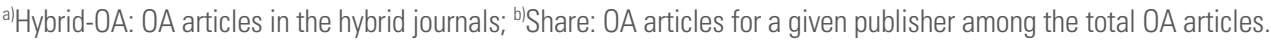

nals, but the gold OA journals published more OA articles than the hybrid journals.

OA publishing trends of the major publishers

To analyze the current status of OA journals and articles published by major publishers, this study defined Hindawi-Wiley journals as Hindawi journals and separated BioMed Central (BMC) from Springer. The top five subscription and OA publishers were selected based on the number of OA articles (Table 3). For 6 years, the top five subscription publishers ac- counted for $30.2 \%$ of all OA articles and the top five OA publishers for $35.3 \%$. Therefore, the 10 major publishers accounted for the majority of OA publishing (65.5\%). Nine publishers, excluding Taylor \& Francis, published more than 10,000 $\mathrm{OA}$ articles annually. Elsevier and Wiley had more hybrid OA articles than gold OA articles. Some of the journals published by the top five OA publishers were mega-journals.

Even though BMC was considered separately, Springer was still the top publisher in JCR with the highest number of OA articles. Scientific Reports and Nature Communications made 
outstanding contributions in this regard. Excluding these journals, Springer had a similar number of OA articles as Elsevier and Public Library of Science (PLoS). Among the 10 selected major publishers, after Springer, the number of OA articles was highest for MDPI, followed in order by BMC, PLoS, and Elsevier. The Royal Society of Chemistry (RSC), which ranked 10th in the total number of articles in a previous study [4], was included in the top five subscription publishers for OA articles, revealing that the RSC made a disproportionate contribution to OA publishing. Elsevier seemed to settle for OA publishing, passively focusing on conditional hybrid OA. Regarding the CAGR for OA articles, PLoS and RSC showed low rates due to the increase in publications by competing journals, while BMC and Hindawi showed relatively low growth rates compared to other publishers. The most active publishers was MDPI, followed in order by Wiley and Frontiers. The overall CAGR for OA articles of the 10 publishers was, on average, $18.0 \%$ over the past 6 years.

\section{Discussion}

Progress towards achieving OA2020

In some recent studies, the proportion of OA articles varied according to the year and database in terms of OA types (Table 4) [5-7]. Unlike other studies, this study investigated the number of OA articles over 6 years, limiting its scope to JCR journals. In JCR 2014 to 2019, the number of OA journals, including hybrid journals, was $80.4 \%$, but the number of OA articles was still small (20.9\%). Gold OA journals published disproportionately many $\mathrm{OA}$ articles relative to their share of journals, but hybrid OA journals showed the opposite tendency. The percentage of OA articles increased gradually, to the point that they now account for more than a quarter of JCR articles.

With respect to the transition of subscription journals to an OA model, which is of interest to librarians, $77.4 \%$ of journals had shifted to hybrid journals, but $22.6 \%$ continued to adhere to the subscription-only model. Despite the very high CAGR for OA articles (25.2\%) in hybrid journals, OA articles ac- counted for only $5.2 \%$, and the rest (94.8\%) were still subscription articles.

Although commercial publishers had actively launched new APC-based gold OA journals and transformed their excellent subscription journals into hybrid journals, considerable progress must still be made for the goal of OA2020 to be achieved. However, the Plan S movement in Europe [8], which encourages research funded by the public grants to be published as OA articles, will accelerate progress towards OA2020.

\section{Characteristics of the major OA publishers}

The top five OA publishers led the growth of OA publishing, accounting for a higher share of OA articles than the top five subscription publishers. In the top five subscription publishers, the gold OA articles' ratio to the hybrid OA articles was 58:42, indicating that the major commercial publishers made efforts for OA publishing. For the past 6 years, the number of gold OA articles was the highest for MDPI, followed in order by Springer, BMC, and PLoS, but the number of gold OA journals was highest for BMC, followed in order by Springer and Elsevier. Meanwhile, PLoS had the highest number of OA articles with the fewest journals. In all JCR journals, the number of articles grew annually at an average rate of $4.7 \%$, but the CAGRs for OA journals and articles was much higher than those of subscription journals and articles. Therefore, OA articles primarily contributed to the recent growth of WoS and JCR. Although many authors publish their articles in gold OA journals due to the relatively inexpensive APCs and rapid publishing, they are not yet willing to pay APCs for hybrid journals.

Limitation: Even though JCR is produced based on WoS, we found differences between these two sources in terms of identifying the OA type of each journal and extracting the number of OA articles from WoS. As of June 2020, there were 1,652 DOAJ-Gold journals in JCR 2019, but 1,796 DOAJGold journals were found in WoS. This study classified 1,640 journals as gold OA journals, which were duplicated in both JCR and WoS. The Korean branch of Clarivate Analytics reported that 7,487 hybrid journals were included in the recent-

Table 4. Comparison of the share of OA articles in several studies

\begin{tabular}{|c|c|c|c|c|}
\hline Study & Investigated DB & OA type & Investigated year & OA share $(\%)$ \\
\hline Open Science Monitor [5] & Scopus with Unpaywall & Gold +hybrid & 2018 & 24.8 \\
\hline Razumova et al. [6] & WoS & Gold + green & 2016 & 28.0 \\
\hline AlRyalat et al. [7] & WoS & Gold + hybrid & 2018 & 24.0 \\
\hline \multirow[t]{2}{*}{ Present study } & JCR with WoS & Gold + hybrid & 2019 & 27.1 \\
\hline & & & 2014-2019 & 20.9 \\
\hline
\end{tabular}

OA, open access; WoS, Web of Science; JCR, Journal Citation Reports. 
ly released JCR 2019, but 7,780 JCR journals had OA articles in WoS. Finally, this study classified 8,369 journals as hybrid journals, including 589 journals with no OA articles in WoS despite their hybrid type. In this study, the OA type of each journal was considered as having existed for 6 years, even if its OA type was changed or it was founded as a new OA journal in 2014 to 2019. Therefore, there may be inconsistencies in each journal's OA type between the period encompassed in the study and 2020. Moreover, the number of hybrid OA articles reflected the WoS search results, but the number of gold $\mathrm{OA}$ articles was determined by analyzing the citable items in JCR. These important limitations should be kept in mind when interpreting this research.

Conclusion: As a result of this study based on JCR 2014 to 2019 , considerable progress must still be made for OA2020 to be achieved. New OA publishers primarily contributed to the increase in OA articles. APC-based gold OA journals are springing up everywhere, giving rise to controversies regarding predatory publishing. Nonetheless, many authors prefer these new gold OA journals because of their rapid publication process compared to traditional journals. OA publishing will have a major influence on stakeholders. Therefore, librarians should take an active interest in OA publishing to appropriately predict and prepare for the library's future in relation to journal services, including subscriptions.

\section{Conflict of Interest}

No potential conflict of interest relevant to this article was reported.

\section{Funding}

The authors received no financial support for this article.

\section{Data Availability}

Data are available from the author upon reasonable request.
Dataset 1. Collected data for OA journals and articles in WoS and JCR 2014 to 2019

\section{References}

1. Max Planck Digital Library. OA2020: a global initiative to drive large-scale transformation of the subscription system [Internet]. Munich: Max Planck Digital Library; 2017 [cited 2020 May 16]. Available from: https://oa2020.org/ wp-content/uploads/OA2020_Conceptual_Framework. pdf

2. Piwowar H, Priem J, Lariviere V, et al. The state of OA: a large-scale analysis of the prevalence and impact of Open Access articles. PeerJ 2018;6:e4375. https://doi.org/10.7717/ peerj.4375

3. Kim SJ, Park KS. Market share of the largest publishers in Journal Citation Reports based on journal price and article processing charge. Sci Ed 2020;7:149-55. https://doi.org/10.6087/ kcse. 210

4. Kim SJ, Park KS. Influence of the top 10 journal publishers listed in Journal Citation Reports based on six indicators. Sci Ed 2020;7:142-8. https://doi.org/10.6087/kcse.209

5. Open Science Monitor. Trends for open access to publications [Internet]. Brussels: European Commission; 2019 [cited 2020 Sep 29]. Available from: https://ec.europa.eu/ info/research-and-innovation/strategy/goals-researchand-innovation-policy/open-science/open-science-monitor/trends-open-access-publications_en

6. Razumova IK, Kuznetsov A. Impact of open access models on citation metrics. J Inf Sci Theory Pract 2019;7:2331. https://doi.org/10.1633/JISTaP.2019.7.2.2

7. AlRyalat SA, Saleh M, Alaqraa M, et al. The impact of the open-access status on journal indices: a review of medical journals. F1000Research [Preprint]. 2019;8:266 [cited 2020 Sep 29]. https://doi.org/10.12688/f1000research.17979.1

8. Kim K. Plan S. Sci Ed 2020;7:78-9. https://doi.org/10.6087/ kcse. 195 